\title{
Cutaneous Manifestations of COVID-19 in Children: Practical Points for Clinicians
}

\author{
Parvaneh Hatami ${ }^{1}$, Hamed Nicknam Asl $^{2}$ and Zeinab Aryanian ${ }^{1,3, *}$ \\ ${ }^{1}$ Autoimmune Bullous Diseases Research Center, Tehran University of Medical Sciences, Tehran, Iran \\ ${ }^{2}$ Department of Dentistry, Rafsanjan University of Medical Sciences, Rafsanjan, Kerman, Iran \\ ${ }^{3}$ Department of Dermatology, Babol University of Medical Sciences, Babol, Iran \\ "Corresponding author: Dermatology Department, Tehran University of Medical Sciences, Tehran, Iran. Email: z_aryanian@yahoo.com
}

Received 2021 December 27; Revised 2022 January 15; Accepted 2022 January 16.

\begin{abstract}
Coronavirus disease 2019 (COVID-19) in previously healthy children is more likely to be mild. However, COVID-19-related cutaneous manifestations in children can be categorized regarding their clinical manifestations. We searched PubMed, Google Scholar, and Scopus in this regard and examined all of the relevant papers published until June 28,2021, with available full-texts. We found various mucocutaneous manifestations after COVID-19 infection and categorized them regarding their etiology: Induced directly by the virus itself or its immunologic response, treatment-related adverse events, post-vaccination rash, and lesions seen as an indirect consequence of the pandemic such as irritant dermatitis resulting from frequent hand washing or stress-induced worsening of some chronic dermatoses such as atopic dermatitis and psoriasis as a result of social isolation. Clinicians should be aware of these manifestations and advocate early isolation while encountering unexplained skin lesions to prevent infection from spreading.
\end{abstract}

Keywords: COVID-19, SARS-CoV-2, Cutaneous Manifestations, Pediatrics, Mucocutaneous Side Effects

\section{Context}

Coronavirus disease 2019 (COVID-19), caused by severe acute respiratory syndrome Coronavirus 2 (SARS-CoV-2), was first reported in Wuhan, China, in December 2019 and soon became a serious public health issue and later a pandemic in March 2020 (1). At the beginning of the pandemic, children were thought to be resistant to infection, and many children with COVID-19 were probably overlooked due to asymptomatic or oligo-symptomatic infection occurring in most children $(1,2)$. Early pediatric patients were reported from China and Italy $(3,4)$ with fever and respiratory symptoms. Although COVID-19 in previously healthy children is more likely to be mild, severe conditions such as multi-system inflammatory syndrome (MIS) were also reported in the pediatric population $(5,6)$.

Based on many previous studies, mainly case reports, cutaneous manifestations are seen in COVID-19, especially in ill adults and asymptomatic children (5). However, their exact prevalence is unknown because of dermatology consultation hesitancy during the pandemic.

\section{Results}

COVID-19-related cutaneous manifestations in children can be categorized regarding their clinical manifestations or etiology: (1) a large group of them are induced directly by the disease: the virus itself or its immune response; (2) some are treatment-related adverse events; (3) some might appear after vaccination against SARS-CoV-2; (4) the rest of them are seen as an indirect consequence of the pandemic, such as irritant dermatitis resulting from frequent hand washing (7) or stress-induced worsening of some chronic dermatoses such as atopic dermatitis and psoriasis (8) as a result of social isolation (9).

Regarding the appearance, cutaneous manifestations can be categorized as acral pseudo-chilblains, urticarial lesions, maculopapular rash, vesicular eruptions, and livedo or necrosis $(10,11)$. Pseudo-chilblain lesions and maculopapular rash on the acral sites are frequently seen in children with COVID-19 $(12,13)$. They were reported as the most common skin lesions by Shah et al., evaluating 149 children with COVID-19 (14).

Moreover, lesions similar to some exanthemas, which have previously been reported to associate with viral infections, were seen in some affected children, such as erythema multiform-like lesions, purpuric eruptions, 
varicella-like lesions, and Gianotti Crosti syndrome $(15,16)$. However, there seem to be some differences in appearance and clinical course in COVID-19 compared to other viral or rheumatologic etiologies that might be used as clinical clues for correct diagnosis. Erythema or maculopapular rash in viral illness generally has a cephalo-caudal distribution and heals with exfoliation, but in COVID-19, it is more likely to be on the acral sites of hands and feet and frequently progress to vesiculation and crusting (14). Moreover, the happening of chilblain-like lesions during the pandemic in the absence of coldness and humidity, positive personal or familial history of Raynaud's phenomenon or autoimmune disorders, and other secondary causes of chilblain strongly lead us to the diagnosis of COVID-19.

A recent study of 50 children hospitalized with COVID19 revealed frequent mucocutaneous manifestations (11). Moreover, patients with mucocutaneous lesions were more likely to have a poor general status and were older than those without skin lesions. Andina-Martinez et al. also found that up to $90 \%$ of patients with MIS had some mucocutaneous lesions (11). Another interesting finding of this study was that unlike patients without the need for admission, skin exanthema in hospitalized children was more likely to appear on the trunk and limbs rather than the acral sites of hands and feet. They suggested that truncal exanthema and conjunctival hyperemia on admission might be considered prognostic factors to predict a higher possibility of the need for pediatric intensive care unit (PICU) admission (11).

Other mucosal lesions reported in the pediatric population consist of pseudomembranous candidiasis, geographic tongue, coated tongue, taste alteration, and hyperemic pharynx (17).

Regarding the etiopathogenesis of cutaneous manifestations, a multicenter study by Carazo Gallego et al. on 62 children with skin manifestations of COVID-19 revealed that chilblains and vesicular lesions were more likely to occur in those with positive SARS-CoV-2 serology and the maculopapular or urticarial rash was more common in patients with negative antibodies (18). This may reflect the different etiopathogenesis of skin rashes in COVID-19. In fact, one can infer that pseudo-chilblain and vesicular lesions are directly linked to antibody formation and can be considered as immunologic-based reactions in comparison to the direct effect of the virus.

Based on the fact that antibody formation needs time, some immunologic-based responses such as Gianotti Crosti syndrome usually appear after the resolution of the disease (19). Hence, assessing the temporal correlation of skin lesions such as chilblain or vesicular rash with the clinical course of COVID-19 might shed more light on the exact pathogenesis of COVID-19-related cutaneous manifestations. Unlike adults $(20,21)$, treatment-related cutaneous lesions are rare in children. However, one case with the temporal correlation of skin lesions with hydroxychloroquine was discussed in the literature (14).

The authors of this study have focused on the immunologic aspects of various topics in dermatology and recently turned their focus on COVID-19-associated dermatologic topics (22-28) as an issue of great importance discussed in this paper. Since the vaccination of children was not started across the world until the last update of this review, vaccine-related cutaneous reactions in children should be dealt with in future studies. However, nonspecific skin rash was seen in four infants whose breastfeeding mothers received a COVID-19 vaccine (29).

\section{Conclusions}

In conclusion, different cutaneous manifestations are seen in children with COVID-19 infection, and clinicians should be aware of them and advocate early isolation while encountering unexplained skin lesions to prevent infection from spreading.

\section{Footnotes}

Authors' Contribution: P. H developed the original idea and the protocol, abstracted and analyzed data, and wrote the manuscript. Z.A and H.N contributed to the development of the protocol, abstracted the data, and prepared the manuscript.

Conflict of Interests: All the authors declare no conflict of interest regarding publishing this manuscript.

Data Reproducibility: The data supporting the findings of this study are available from the corresponding author upon reasonable request.

Funding/Support: We received no funds for this project.

\section{References}

1. Munro APS, Faust SN. COVID-19 in children: Current evidence and key questions. Curr Opin Infect Dis. 2020;33(6):540-7. doi: 10.1097/QCO.0000000000000690. [PubMed: 33027185].

2. Khalili M, Iranmanesh B, Mohammadi S, Aflatoonian M. Cutaneous and histopathological features of Coronavirus disease 2019 in pediatrics: A review article. Dermatol Ther. 2021;34(1). e14554. doi: 10.1111/dth.14554. [PubMed: 33210417]. [PubMed Central: PMC7744858].

3. Coronaviridae Study Group of the International Committee on Taxonomy of Viruses. The species Severe acute respiratory syndromerelated coronavirus: classifying 2019-nCoV and naming it SARS-CoV2. Nat Microbiol. 2020;5(4):536-44. doi: 10.1038/s41564-020-0695-Z. [PubMed: 32123347]. [PubMed Central: PMC7095448].

4. World Health Organization. Coronavirus disease (COVID-19) pandemic. Geneva, Switzerland: World Health Organization; 2022, [updated 2022; cited 19th Jun 2020]. 
5. Recalcati S. Cutaneous manifestations in COVID-19: A first perspective. J Eur Acad Dermatol Venereol. 2020;34(5):e212-3. doi: 10.1111/jdv.16387. [PubMed: 32215952].

6. Hutton B, Salanti G, Caldwell DM, Chaimani A, Schmid CH, Cameron C, et al. The PRISMA extension statement for reporting of systematic reviews incorporating network meta-analyses of health care interventions: Checklist and explanations. Ann Intern Med. 2015;162(11):777-84. doi: 10.7326/M14-2385. [PubMed: 26030634].

7. Patruno C, Fabbrocini G, Stingeni L, Napolitano M. The role of occupational dermatology in the COVID-19 outbreak. Contact Dermatitis. 2020;83(2):174-5. doi: 10.1111/cod.13568. [PubMed: 32319103]. [PubMed Central: PMC7264514].

8. Garcovich S, Bersani FS, Chiricozzi A, De Simone C. Mass quarantine measures in the time of COVID-19 pandemic: Psychosocial implications for chronic skin conditions and a call for qualitative studies. J Eur Acad Dermatol Venereol. 2020;34(7):e293-4. doi: 10.1111/jdv.16535. [PubMed: 32330329]. [PubMed Central: PMC7267356].

9. Bodak N, Chiaverini C, Barbarot S. COVID-19 lockdown induced acral dermatosis in children.J Eur Acad Dermatol Venereol.2020;34(12):e75860. doi: 10.1111/jdv.16797. [PubMed: 32594634]. [PubMed Central: PMC7361382].

10. Galván Casas C, Català A, Carretero Hernández G, Rodríguez-Jiménez P, Fernández-Nieto D, Rodríguez-Villa Lario A, et al. Classification of the cutaneous manifestations of COVID-19: A rapid prospective nationwide consensus study in Spain with 375 cases. Br J Dermatol. 2020;183(1):71-7. doi: 10.1111/bjd.19163. [PubMed: 32348545]. [PubMed Central: PMC7267236].

11. Andina-Martinez D, Nieto-Moro M, Alonso-Cadenas JA, Anon-Hidalgo J, Hernandez-Martin A, Perez-Suarez E, et al. Mucocutaneous manifestations in children hospitalized with COVID-19. J Am Acad Dermatol. 2021;85(1):88-94. doi: 10.1016/j.jaad.2021.03.083. [PubMed: 33819537]. [PubMed Central: PMC8017917].

12. Roca-Gines J, Torres-Navarro I, Sanchez-Arraez J, Abril-Perez C, SabalzaBaztan O, Pardo-Granell S, et al. Assessment of Acute Acral Lesions in a Case Series of Children and Adolescents During the COVID-19 Pandemic. JAMA Dermatol. 2020;156(9):992-7. doi: 10.1001/jamadermatol.2020.2340. [PubMed: 32584397]. [PubMed Central: PMC7317654].

13. Colmenero I, Santonja C, Alonso-Riano M, Noguera-Morel L, Hernandez-Martin A, Andina D, et al. SARS-CoV-2 endothelial infection causes COVID-19 chilblains: Histopathological, immunohistochemical and ultrastructural study of seven paediatric cases. Br J Dermatol. 2020;183(4):729-37. doi: 10.1111/bjd.19327. [PubMed: 32562567]. [PubMed Central: PMC7323219].

14. Shah S, Akhade K, Ganguly S, Nanda R, Mohapatra E, Goel AK. Cutaneous manifestations associated with COVID-19 in children: A systematic review. J Family Med Prim Care. 2021;10(1):93-101. doi: 10.4103/jfmpc.jfmpc_1389_20. [PubMed:34017709]. [PubMed Central: PMC8132763].

15. Marzano AV, Genovese G, Fabbrocini G, Pigatto P, Monfrecola G, Piraccini BM, et al. Varicella-like exanthem as a specific COVID-19associated skin manifestation: Multicenter case series of 22 patients. J Am Acad Dermatol. 2020;83(1):280-5. doi: 10.1016/j.jaad.2020.04.044. [PubMed: 32305439]. [PubMed Central: PMC7161488].

16. Olisova OY, Anpilogova EM, Shnakhova LM. Cutaneous manifestations in COVID-19: A skin rash in a child. Dermatol Ther. 2020;33(6). e13712. doi: 10.1111/dth.13712. [PubMed: 32472593]. [PubMed Central: PMC7300520].

17. Bardellini E, Bondioni MP, Amadori F, Veneri F, Lougaris V, Meini A, et al. Non-specific oral and cutaneous manifestations of Coronavirus Disease 2019 in children. Med Oral Patol Oral Cir Bucal.2021;26(5):e549-
53. doi: 10.4317/medoral.24461. [PubMed: 33772567]. [PubMed Central: PMC8412440].

18. Carazo Gallego B, Martin Pedraz L, Galindo Zavala R, Rivera Cuello M, Mediavilla Gradolph C, Nunez Cuadros E. Skin lesions in children during the first wave of the SARS-CoV-2 pandemic. Med Clin (Engl Ed). 2021;157(1):33-7. doi: 10.1016/j.medcle.2021.03.007. [PubMed: 34151022]. [PubMed Central: PMC8196301].

19. Swali RN, Lee EB, Adams JL. Gianotti-crosti syndrome in the setting of recent coronavirus disease-19 infection. Pediatr Dermatol. 2021;38(3):629-31. doi: 10.1111/pde.14518. [PubMed: 33641205]. [PubMed Central: PMC8013985].

20. Seirafianpour F, Sodagar S, Pour Mohammad A, Panahi P, Mozafarpoor S, Almasi S, et al. Cutaneous manifestations and considerations in COVID-19 pandemic: A systematic review. Dermatol Ther. 2020;33(6). e13986. doi: 10.1111/dth.13986. [PubMed: 32639077]. [PubMed Central: PMC7362033].

21. Najar Nobari N, Seirafianpour F, Mashayekhi F, Goodarzi A. A systematic review on treatment-related mucocutaneous reactions in COVID19 patients. Dermatol Ther. 2021;34(1). e14662. doi: 10.1111/dth.14662. [PubMed: 33301232]. [PubMed Central: PMC7883102].

22. Balighi K, Hatami P, Sheikh Aboli MJ, Daneshpazhooh M, Ghiasi M, Mahmoudi HR, et al. Multiple cycles of rituximab therapy for pemphigus: A group of patients with difficult- to-treat disease or a consequence of late rituximab initiation? Dermatol Ther. 2021. e15249. doi: 10.1111/dth.15249. [PubMed: 34910350].

23. Al Bazzal A, Hatami P, Abedini R, Etesami I, Ayanian Z, Ghandi $\mathrm{N}$. A prospective comparative study of two regimens of diphenylcyclopropenone (DPCP) in the treatment of alopecia areata. Int Immunopharmacol. 2021,101(Pt B):108186. doi: 10.1016/j.intimp.2021.108186. [PubMed:34710658].

24. Aryanian Z, Shojaee N, Goodarzi A, Shirzadian A, Hatami P. Influence of Narrow-Band Ultra Violet B Phototherapy on Homocysteine Status: A Prospective Study of Patients with Different Cutaneous Disorders. Photobiomodul Photomed Laser Surg. 2021;39(9):607-11. doi: 10.1089/photob.2021.0025. [PubMed: 34546111].

25. Ghandi N, Daneshmand R, Hatami P, Abedini R, Nasimi M, Aryanian $\mathrm{Z}$, et al. A randomized trial of diphenylcyclopropenone (DPCP) combined with anthralin versus DPCP alone for treating moderate to severe alopecia areata. Int Immunopharmacol. 2021;99:107971. doi: 10.1016/j.intimp.2021.107971. [PubMed: 34298402].

26. Aryanian Z, Balighi K, Daneshpazhooh M, Karamshahi E, Hatami P, Goodarzi A, et al. Rituximab exhibits a better safety profile when used as a first line of treatment for pemphigus vulgaris: A retrospective study. Int Immunopharmacol. 2021;96:107755. doi: 10.1016/j.intimp.2021.107755. [PubMed: 34162136].

27. Torkashvand F, Rezaeian M, SheikhFathollahi M, Mohammadreza Khaninezhad S, Hatami P, Bidaki R. [A survey on psychiatric disorders in patients improved cutaneous leishmaniasis in the city of Rafsanjan in 2014]. J RafsanjanUniv Med Sci. 2016;14(10):879-94. Persian.

28. Hatami P, Balighi K, Nicknam Asl H, Aryanian Z. COVID vaccination in patients under treatment with rituximab: A presentation of two cases from Iran and a review of the current knowledge with a specific focus on pemphigus. Dermatol Ther. 2022;35(1). e15216. doi: 10.1111/dth.15216. [PubMed: 34811862].

29. McMahon DE, Amerson E, Rosenbach M, Lipoff JB, Moustafa D, Tyagi A, et al. Cutaneous reactions reported after Moderna and Pfizer COVID19 vaccination: A registry-based study of 414 cases. J Am Acad Dermatol. 2021;85(1):46-55. doi: 10.1016/j.jaad.2021.03.092. [PubMed: 33838206]. [PubMed Central: PMC8024548]. 